\title{
RELATING TRADITION TO INNOVATION WITHIN CHINESE ART
}

\author{
Lijia Ke \\ Thames Valley University \\ 23 West Hill Road \\ Brighton \\ East Sussex \\ Ke_lijia@yahoo.co.uk \\ http://www.creativetechnology.org.uk/lijia/
}

\begin{abstract}
Digital technology brings great impact to art creation as well as a gap between traditional art and modern society. Finding the link between traditional and the modern has been a focusing issue in some countries such as China. In order to make a contribution to this process, this study focuses on the potential of digital media in the context of traditional visual art, in particular on Chinese literati painting. This helps to create the connection between the older idea and more modern thinking.
\end{abstract}

\section{TRADITIONAL ART CONCEPT IN A DIGITALISE ERA}

Every art form emerges relying on the development of society and technology at the time. Digital arts appeared along with the development and popularity of computer technology. Although the earliest digital artwork can be dated back to the 1960s, it was after the Apple Computer Company invented the Macintosh desktop publishing (DTP) system in the 1980s, that the digital arts field started to gain more and more attention. [1]. The development of digital technology drove more artists to integrate their artistic talent with digital techniques and depart from the conventional disciplines to use computer-based technology while computer-aided design (CAD) and computer-aided manufacturing (CAM) became more developed and popular. While digital technology is showing its ability to support industrial and commercial design, its unique visual presentation language has also been adopted in some artists' art works.

Digital technique brings excitement and visual novelty to artists. At the same time, because of cultural and technical differences, it shows some disadvantages in showing the same expression and emotion as the traditional visual arts, such as paintings. There are some misconceptions in both digital art and in Chinese traditional art, and because of this, it seems that digital arts can only work for certain themes and have no relationship to traditional art concepts. Through study of the cultural origins of traditional art, the potential for developing visual presentation and a sense of the aesthetic in the digital arts, as well as forming a bridge with the traditional arts, can be defined by adopting some fundamental delineations, colours, and cultural elements such as painting objects with recognised meanings. Using traditional arts, especially the literati art concept to enrich digital artwork content and taking into account the tradition of literary achievement to fill the cultural gap in relation to the digital arts, these are the most logical ways of presenting Chinese traditional beauty in cyberspace. As the title of 
the paper shows, Chinese is the cultural background of the study, and so terms such as tradition or art refers to Chinese tradition or art unless specify otherwise.

The difference in the viewers' emotional response to traditional art, as compared to the digital, firstly comes from the difference in cultural origins. Taking painting as an example, traditional paintings are painted based on the depth of cultural and historical sources, which contain elements of both regional and national cultures. Traditional arts have many different forms; they contain a depth of cultural accumulation and histories; they reflect to a general, possibly nationwide psychological sensibility, customs, aesthetic outlook and aesthetic sentiment. The deep historical traditions, numerous style schools, huge amounts of regional cultural elements, as well as the form and cultural origins of the traditional arts, bring affinity and resonance to people psychologically. On the other hand, as a new art form, digital art was developed in a technological and manufacturing environment. Its aesthetic emotion has a lack of traditional culture base. The limitation of themes and presentation formats makes it seem unfamiliar and distant to some people. Secondly, the modern technologies involved cause differences in the approaches to the methods of creation and presentation. Apart from material and skill developments, techniques of traditional arts such as paintings have never faced such a big impact as that created by digital technology. As people still generally think that traditional culture only exists within traditional craft material and the traditional skills, it takes time for modern technology such as digital technique to effect the transformation and for its place to be recognised and accepted. A long history of traditional technique and ink style in paintings makes people accept the artistic value of traditional paintings. However, lack of understanding and receptivity of the digital arts cause an emotional distance for viewers.

The digitalised graphic process is the keystone of digital art creation technology. As an important component, a vector graph presents images in 'hard edge' style which is suitable for graphic design, such as logo and standard font images. However, this 'hard edge' creates a cold and machine-like style. Bitmap (also known as Raster) is other major 2D graphic type for digital design, which is made up of pixels in a grid. Again, because of the requirement of digital technology, visual sense colours have to be transformed into digits. These digitalised neat and accurate visual styles make digital works seem to lack connection with traditional culture and artistic languages, which is also the defect of many contemporary digital art works. Moreover, the style of presentation is another factor that could cause different emotional reactions. Traditional painting went through a long period of development and transmutation, which brought into being many presentational forms and styles. These traditional artistic languages are easy to communicate with, as well as to create resonance with viewers. On the other hand, machine-like digital creation comes from different mode of thinking and causes disadvantage in presenting boarder and deeper meaning in Chinese philosophical sense. Take the 2008 Beijing Olympia candidature logo design (figure 1) as an example, which is considered to be one of the most successful logo designs in China. It was created followed the theme of combining a traditional art style and modern digital technique. It took Han Meilin's (1936-) hand painted style to enhance Chen Shaohua's (1954-) 
digital graphic creation, which provides a good example of using the essence of traditional art to enrich digital art creation.

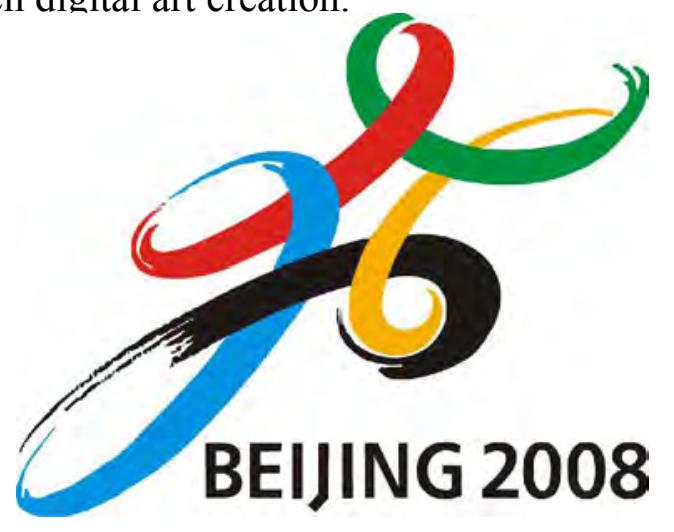

Figure 1

The winning point of this logo design is adopting cultural elements that include the shape of the Chinese knot (also called 'knot of one heart'), an association with philosophy of Taiji, and brush painting/writing visual style. Absorbing different culture elements and knowledge from other areas for the presentation of digital art is a way to develop digital arts. Integrating national transcendence traditional culture and art modalities is a way to enhance the charm and vitality of digital art design. Digital arts creation also has its own attractive quality. It provides a visualised approach for artists' creative constructs as well as maximising the assimilation of artists' thought and imagination into their art works. After people have overcome the initial wonderment of the abilities of digital technology, the question of the value of digital art remains.

Just as Western painting went through classicism, realism, impressionism, abstract expressionism and so on since the Renaissance, Chinese paintings have also been through many schools of transmutation in the past. Change in an art form or style can be a long process with a complex background. Just as what many art forms have been through in history, although most people have accepted digital art as an individual art form, the argument about its value remains. Because of the commercial background of digital art design, some people perceive it superficially and without understanding. There are three major erroneous views about digital arts. The first one is overrating the ability of computer technology. Considering the computer as an all-powerful tool is a deification, and an exaggeration of the power of digital technology. It is misleading for the discipline of creating digital art and its form of presentation. Taking simulation instead of original creation is a naive understanding of the relationship between digital simulative and creative abilities. Being aware of the expressive character of digital art creation and recreation is the way to use it to its biggest advantage. A second misunderstanding is 'commercial purpose'. As computer design has been widely used in commercial industries, the concept of computer arts seems to be lacking a grounding in art theory. Many computer art works are showing personal computer skill rather than an artistic outlook. From some articles and interviews of digital creation, such as When video picture meets traditional culture, Interview Xu Yi, Huang Ying's Pond and Pond 
Rhyme, circle techniques and methods used in creating artworks [2]. Art and culture have not been demonstrated in the digital world for long. Until now, it has been hard for many people to put digital art on to a higher artistic aesthetics plane. Along with developing digital arts and innovating its mode of creation, its artistic value will break the commercial impression and occupy a more important place in the realm of art. The third misunderstanding is the value of digital arts. Traditional arts are valued and more accepted for their historical sources, regional customs and their abilities to deliver certain artistic concepts. Compared to traditional art works, digital technology's mass production ability becomes a disadvantage in an artwork valued from psychological point of view. However, we should not recognise digital art creation as merely one kind of technology, but a new form of art, which has its own visual language and presentation style. Therefore, the value of digital art works should not be measured by using traditional values.

Moreover, although the computer is also called 'electronic brain' in some languages, it still does not function in the same way as a human brain does. As with other art tools, the computer can be seen as an extension of the human brain, where the concept of an artwork emerges. There is no such thing as the 'perfect' form of art. The visual results that digital technology can bring to us are various. However, there are also disadvantages in terms of computer arts. The meaning of an artwork quite often depends on the cultural or educational background of the artist or viewer. The study takes culture and education as the premise of creating in order to make an innovation in artistic expression in computer art. Cultural connection in this study is the determining factor for creating a good piece of artwork. Lack of cultural and educational background is the current reason that computer art has less value in the Chinese fine arts field. Through this study, a new meaning for computer art may be demonstrated.

Showing educational background is considered to be the most important thing in Chinese literati art. Working out the connection between digital art and traditional artistic sources is one way to enrich the language of digital art and link the traditional and the modern in China. While many artists have incorporated current computer technology into their work to enhance their artistic visions - pushing the boundaries of their respective disciplines into the digital medium, this study focuses on making an extension of Chinese literati art into this new medium.

\section{TRADITIONAL ART CONCEPT IN A DIGITALISE ERA}

The relationship between tradition and innovation is not a new topic in China. Chinese artists have been making a variety of attempts in combining studying tradition and making innovation for over a century. After the modernism popularity, a 'neoliterati painting' trend became popular in the middle of 1980s. Those paintings with high attention in traditional style and 'brush and ink' ${ }^{\text {' }}$ skill illustrated that Chinese painters have turned from anti-tradition attitude to reconsidering their artwork development with their background. However, this so called neo-literati painting practice cannot be considered to be successful, because it is simply renews the style and

\footnotetext{
1 'Brush and ink' should be taken as one word which applies to the painting materials and instruments for traditional Chinese painting.
} 
form of old literati paintings, which should be considered as refashioning literati painting. Brush and ink are very important in Chinese traditional painting principle, as they embody a concentrated reflection of visual character of Chinese painting. However, the brush and ink should not be perceived as a kind of skill or a form of method. More important is to understand its spirit which is its philosophical background. Only with this spirit, a painting can be recognised as literati painting. The spirit of literati painting is to pursue and develop visual art with literary comprehension. And from that, literati painting can be seen as the most ethos representative art form within Chinese traditional art. For literati painting, the whole literati spirit of traditional Chinese culture can be referenced as its spiritual source. Literati painting is the most glorious chapter in Chinese painting history. It reflects Chinese literatures, philosophies, and history, as well as an aesthetic standard which includes showing a painter's personality and morality. Because of its various functions, it is still the mainstream of Chinese painting today, even though scholars no longer occupy the highest level of the society. With the important position that the literati painting occupies in Chinese society, inviting it into the digital world has great significance for Chinese people.

When digital technology shows its ability in producing high-end products as well as in people's daily life, some art practitioners consider that digital technique is the core of the creation. It is just as Theodore Roszak (1933- ) suggested that, perhaps, like the unfortunate emperor, the computer has been overdressed with false claims made by those with something to gain by it. He raised the question:

Is our capacity to think creatively being undermined by the very 'information' that is supposed to help us? Is information processing being confused with science or even beginning to replace thought? And are we in danger of blurring the distinction between what machines do when they process information and what minds do when they think? [3]

For this concern, the best answer might be that "technology is the engine and literae humaniores is the wheel" [4]. The reason that digital technology seems practical is not only because it is capable of achieving good visual quality but it can also be utilised by anyone for multiple purposes. How to treat and evaluate the impact that high technology brings to culture is a question that bothers many artists and art appreciators. Ignore the differences between computer and brush; they are just media standing between artists and their works after all. Media are just the physical ministration of the art work; it does not represent the entire nature of art. Digital art cannot be seen as a pure technological achievement, nor perceived as a new technique. While visual beauty may be achieved, the more important thing is that the internal beauty is also presented.

In literati painting, internal beauty refers to the philosophical idea within the artwork. Literati painting was started by scholars who were not professional painters but interested in art, and who used painting to present their understanding and belief, which they gained through study and life. For this purpose, the painting skill became less important than the concept, while colour, layout and painted objects played important roles in reflecting certain philosophical or moral ideas. When the spirit is the most 
important thing in the artwork, it is not too hard for Chinese to accept applying the same concept to a different artistic medium.

The artistic concepts of literati painting have been applied to other professions, such as garden design, interior design and novel writing. Since the last century, this extension can also be found in caricature, animation, commercial graphic design and education. The power of this extension did not come from literati painting but from thousands years of literature. On the other hand, the visual expression in literati paintings in return made a great contribution to the continuity and popularity of these philosophy and literature. Since the late $19^{\text {th }}$ century, brush and ink painting style started to face the challenge from other painting styles, such as oil painting and watercolour painting [5]. 'Painting technique' then became the core argument for nearly a century. This argument includes two aspects. One aspect is which technique or method has more advantage. The other aspect is that whether the technique or skill should be the main focus for creating an artwork. The main reason that this argument is still going in Chinese painting society is because the concepts of literati painting have never been made clear.

While some people are still fighting for the position of 'brush and ink', some artists such as Liu Guosong (1932- ) and Wu Guanzhong (1919- ) suggest that artists and viewers should pay more attention to the artistic outcome rather than the method of creating the work. Originally 'brush and ink' was just one kind of method for painting [5]. However, some people take 'brush and ink' skill as the only standard by which to judge the painting, that is putting the incidental before the fundamental. Although skill in handling 'brush and ink' can help artists to express themselves in a certain way, it should not be used to restrict the method of creating literati art. Just as Wu Guanzhong argued: "Method should be something to serve my idea and my artwork. New method should not be rejected if it can coordinate with the need of my work" [6].

In order to find a place for digital art in the 'literati art' realm, the research part of this study has involved art history, the concept of traditional art, as well as some case studies. Since there are no clear guidelines for creating literati art, this study clarifies the concepts and spirit of literati art from the history and philosophical background of each component of literati painting. A multimedia project has also been created to demonstrate the idea and examine the theory that the study proposes, which is the possibility of presenting literati art concepts with new presentation technique. From this practice, the aim of the study is not only to achieve continuity in relation to tradition of art concepts but also to demonstrate a new stage for both Chinese literati art and digital art.

\section{References}

[1] PAUL, Christiane. Digital art. London : Thames and Hudson, 2003. p.8-11.

[2.1] ZHAO YING. When video picture meets traditional culture. Digital Age, 2007, $8^{\text {th }}$ issue.

[2.2] ANGELA, Interview $\mathrm{Xu} \mathrm{Yi}$ : traditional nation always brings innovation in beauty. Global Digital Magazine,

[2.3] Jiangsu Channel of Xinhua Net, $27^{\text {th }}$ May 2004, Yi Ming, Huang Ying's 'Pond' and 'Pond Rayme', BTV, 27 Oct 2005 
EVA 2009 London Conference 6-8 July

Lijia Ke

[3] ROSZAK, Theodore. The cult of information: the folklore of computers and the true art of thinking, University of California Press, 1994.

[4] WENBIAO, Cao. Science is the engine, literae humaniores (culture) is the wheel. Guangming Daily Press, Theory Section, 30 August 2003.

[5] YUANPEI, Cai. Speech for Beijing University Painting Technique Association. Beijing University Monthly Publication, 25 Oct 1919.

[6] GUANZHONG, Wu. Brush and ink equal to zero. Mingpao Weekly, 1992.

[7] GUANZHONG, Wu. Why I said 'Brush and Ink Equal to Zero'. Guangming Daily, 7 April 1999. 